Voix et Images

\title{
Une affinité entre l'auteur et le réalisateur, entrevue avec Guy Dufresne
}

\section{Jacques La Mothe}

Volume 9, numéro 1, automne 1983

Guy Dufresne

URI : https://id.erudit.org/iderudit/200419ar

DOI : https://doi.org/10.7202/200419ar

Aller au sommaire du numéro

Éditeur(s)

Université du Québec à Montréal

ISSN

0318-9201 (imprimé)

1705-933X (numérique)

Découvrir la revue

Citer ce document

La Mothe, J. (1983). Une affinité entre l'auteur et le réalisateur, entrevue avec Guy Dufresne. Voix et Images, 9(1), 29-37. https://doi.org/10.7202/200419ar d'utilisation que vous pouvez consulter en ligne.

https://apropos.erudit.org/fr/usagers/politique-dutilisation/ 


\title{
Une affinité entre l'auteur et le réalisateur, entrevue avec Guy Dufresne
}

\author{
par Jacques La Mothe, Université du Québec à Montréal
}

V.I. Qu'est-ce qui vous a amené à écrire pour la radio et la télévision beaucoup plus que vous ne l'avez fait pour le théâtre par exemple?

G.D. Ce sont les événements : vers l'âge de vingt ans, pour des raisons de santé, je n'ai pu m'inscrire à l'Université après mon cours classique. Les médecins m'ont recommandé la campagne. Je suis devenu pomiculteur. À Frelighsburg, plus précisément. Je m'étais dit que, tout en exerçant ce métier, j'écrirais. Du thêâtre. Mais le théâtre que j'écoutais c'était surtout celui de la radio. J'étais fasciné, dans le temps, par Claude-Henri Grignon, Robert Choquette, Yves Thériault, Félix Leclerc. Je me disais : «Bon Dieu que ça me tenterait». J'avais le goût d'écrire mais il fallait que ce soit en même temps un gagne-pain : j'étais obligé de gagner ma vie. Les revenus de la pomiculture sont aléatoires, c'est difficile d'être pomiculteur parce qu'il faut être indépendant de fortune et je ne l'étais pas... Une certaine année, les fleurs ont gelé. Un printemps anarchique, catastrophique! Cela signifiait concrètement que je n'avais plus de revenu. Or cette même année, Radio-Canada lançait son premier concours radiophonique : j'y ai participé, et j'ai gagné (avec le Contrebandier, en 1946)!

C'est à la suite de cet événement que j'ai rencontré Guy Maufette; on cherchait un auteur pour une série qui s'appelait pompeusement les «Fondateurs de l'Église canadienne». Il s'agissait d'évoquer, d'imaginer le pays dans les années 1600 , de faire le portrait des grandes figures de l'Histoire, d'aller au fond de ces personnages mystiques, de les présenter dans toute leur complexité sans pour autant les placer sur un piédestal. Je devais, au contraire, les plonger dans la vie du temps. J'ai adoré ça!

C'est ainsi que ma carrière a débuté. L'émission s'intitulait le Ciel pardessus les toits. Elle a duré huit ans (du 12 octobre 1947 au 24 avril 1955) de sorte que, pendant la saison active, la saison de pomiculture, je cultivais mes pommiers alors que, pendant la saison morte j'écrivais 
quelque vingt à vingt-cinq textes d'une demi-heure. Puis la télévision est venue. Cela m'a donné l'occasion de faire le saut. J'ai écrit la série Cap-aux-Sorciers (du 7 juin 1955 au 12 juin 1958) réalisée par Paul Blouin. Une belle expérience. Pourtant, ce que je voulais faire, c'était du théâtre! Si j'avait été à la ville, proche des gens de théâtre, peut-être aurais-je fait comme Marcel Dubé qui utilisait la radio comme revenu d'appoint mais dont la principale activité était le théâtre.

V.I. À l'époque de votre cours classique au collège Brébeuf, n'y a-t-il pas eu des ceuvres ou des gens qui vous ont marqué?

G.D. Quand j'étais au collège, avant les Belles-Lettres, j'étais endormi, engourdi, frêle et nerveux. J'avais besoin de bouger beaucoup après les classes. Je ne lisais pas. Je faisais du sport. Je n'avais aucun goût pour la vie intellectuelle. J'ai eu le coup de foudre en Belles-Lettres avec François Hertel. Il nous a fait lire une multitude d'œuvres qui, à cette époque (1932-1933), étaient presque interdites : c'est en cachette qu'on lisait Verlaine ou Baudelaire! Ce fut une révélation. J'ai cessé de me dépenser physiquement pour me plonger corps et âme dans la lecture. En Rhétorique, j'avais parcouru assez de terrain pour m'apercevoir que j'étais fou du théâtre. C'était l'époque des spectacles du Père Legault à Saint-Laurent. J'adorais. Surtout les discussions qui suivaient le spectacle. Quand il a créé les Compagnons de SaintLaurent (1937-1952), j'étais tout à fait disposé à faire partie de la troupe mais mon père qui était médecin ne voulait pas. J'ai raté le bateau contrairement à d'autres qui ont fait carrière soit comme acteurs (Jean-Louis Roux, Jean Coutu) soit comme réalisateurs (Claude Désorcy; Jean-Paul Fugère).

V.I. La fonction de dramaturge n'existait pas en pratique, dans notre société.

G.D. Il n'y en avait pas tellement parce que le mouvement de la radio et de la 'télévision n'a pris une véritable ampleur qu'au cours des années cinquante. Il y avait bien quelques écrivains qui avaient commencé à écrire pour la radio mais on ne les prenait pas très au sérieux, c'étaient de drôles d'hurluberlus!

Quant à moi j'ai découvert ma passion pour le théâtre en BellesLettres, grâce à un excellent jeune professeur de grec, Marc Patry, qui nous avait fait étudier et traduire Antigone. Il nous avait demandé de dépeindre le caractère des personnages. Il avait été étonné de la qualité et de la profondeur de mon travail.

V.I. Vous avez découvert une motivation, une aptitude?

G.D. Certainement. Une aptitude et un goût pour le théâtre. Et même si, à la fin du cours classique, $\mathrm{j}$ 'ai dû me réfugier à la campagne pour cause de maladie, Antigone en grec est resté pour moi comme le symbole, le 
prototype de la perfection. J'ai toujours voulu atteindre la qualité d'Antigone, produire des ceuvres qui échappent au temps. Aujourd'hui encore je relis la pièce avec la même émotion; chaque ligne est là, c'est fort, chaque mot porte.

V.I. Existe-t-il une collaboration distincte voire spécifique entre l'auteur et le metteur en scène, entre le dramaturge et le réalisateur de la radio ou de la télévision; cela dépend-il des individus? Leur implication face à l'élaboration d'une œuvre en train de s'accomplir, est-elle aussi approfondie?

G.D. Que ce soit à la radio, à la télévision ou au théâtre, on pourrait voir les différences, mais il y a certainement quelque chose en commun. Pour qu'une ouvre prenne vie et touche profondément les gens, il faut au point de départ qu'entre l'auteur et le réalisateur ou le metteur en scène, il y ait une communion, une profonde complicité. La réussite en ce domaine dépend de l'échange riche et constant entre l'auteur et le réalisateur.

À la radio, Guy Mauffette ne participait pas à la conception; c'est moi qui, à la campagne, créait le texte seul. Par ailleurs, quand on se rencontrait, on avait tellement à dire que les heures manquaient, on $s$ 'entendait et j'avais beau truffer un texte de difficultés, Guy en saisissait toutes les nuances, il comprenait tout : quand on disait «les deux Guy», on avait tout dit! Il faut dire aussi que la radio est un media merveilleusement simple, les comédiens ne sont pas obligés de mémoriser; une émission d'une demi-heure peut se faire en trois heures. C'est à l'auteur de créer une ouvre de valeur; au réalisateur de saisir tout ce qu'il a mis dedans et de choisir les bons interprètes. Dès qu'on écoute une grande réalisation faite par un grand réalisateur, le comédien disparaît quasiment et c'est le personnage qui ressort : ce n'est plus Huguette Oligny, c'est Marie de l'Incarnation. L'interprète donne sa pleine mesure parce qu'il a été bien dirigé. De ce point de vue Guy Mauffette était un réalisateur aux ressources inouies.

V.I. Vous avez fait le pas de la radio à la télévision. Personne ne savait très exactement comment le media fonctionnait : l'utilisation de cette machine énorme était, jusqu'à un certain point, expérimentale.

G.D. À la radio, on avait appris sur le tas. Robert Choquette avait déblayé le terrain. C'est lui qui nous avait donné les bases, les grandes lignes, enseigné l'ABC. Lorsque Radio-Canada avait institué le concours d'écriture radiophonique, Choquette avait donné, pour l'occasion, en tranches d'une demi-heure, tous les dimanches (en 1951), sept ou huit leçons qu'il avait qualifiées de "Catéchisme du radio-dramaturge». Je me rappelle qu'au début, je me sentais démuni comme quelqu'un sans marteau qui veut planter un cloú. Il avait constitué une grammaire, nous avait donné des outils. Il avait tout synthétisé : comment faire les 
transitions, comment établir le choix des personnages. Si c'était de l'Histoire, comment choisir une langue qui nous transporte dans un autre milieu, dans un autre lieu, comment nous documenter sans crayon c'est-à-dire en revivant de l'intérieur les drames et les événements de manière à mieux pouvoir les rendre par une parole qui soit juste : «Si vous voulez que ce soit vivant, plausible dans la bouche des gens, vous avez, à moins que ce soit une langue de convention, plus d'un choix : il y a quinze, vingt manières d'écrire une chose. Tâchez d'écrire quelque chose qui se dise bien parce que les gens l'écoutent».

V.I. Donc beaucoup plus qu'une analyse de type intellectuel, une observation plurisensorielle, qui permet en quelque sorte de rejouer ce qui a été perçu?

G.D. C'est ça! Ecouter parler les gens, les regarder faire, observer, toujours observer. Pour qu'un texte soit plausible, il faut qu'il soit travaillé. Le contact avec le réel est absolument nécessaire. Il faut rester branché sur la vie. D'ailleurs pour écrire, il faut se documenter, puis situer les personnages, les découvrir, les créer, ce qui est une chose élémentaire mais qui s'apprend; ensuite bâtir une intrigue en ayant le souci de toujours précéder le spectateur. Il faut aussi, bien sûr, faire l'apprentissage de l'écriture, maîtriser l'art d'un bon début, d'une trame, d'un dénouement. Si à la radio on avait pu bénéficier de l'enseignement de Robert Choquette, à la télévision il n'y a pas eu de professeur : c'est en expérimentant qu'on a appris. Cap-aux-Sorciers n'aurait pu être écrit sans le support de Paul Blouin et d'Aimée Arsenault-Cacopardo. Pour chaque émission, nous nous réservions tout un après-midi, où tous les trois nous causions : moi, je jetais en vrac toutes les idées que j'avais en tête et puis là on bâtissait l'émission : toute la série a été élaborée de cette manière.

V.I. Vous n'arriviez donc pas avec un scénario écrit. Et si vous aviez en tête un canevas qui englobait le sujet de plusieurs émissions, c'était plutôt des idées qu'ensemble vous mettiez en forme, ce qui devait demander une disponibilité particulière de la part des concepteurs.

G.D. Aucun texte écrit, sauf quelques notes sur du papier. mais surtout un flot d'idées en tête : qu'est-ce qu'on fait avec chacun des personnages cette fois-ci? Un feuilleton était, selon moi, une émission où les personnages évoluaient. Je ne voulais pas qu'au bout d'un an les personnages fassent la même chose qu'au début. Je ne voulais pas que ce soit un téléroman mais un feuilleton : un téléroman c'est une "capsule» qui n'a que peu ou pas de rapport avec les émissions précédentes et celles qui suivent. À la radio, les feuilletons étaient quotidiens. L'auditeur avait hâte de savoir ce qui allait arriver le lendemain. C'est ce que je voulais faire à la télévision.

V.I. Vous avez conservé cet héritage de la radio? 
G.D. Robert Choquette nous avait incités à terminer une émission en donnant le gout aux gens de voir ce qui viendrait après. Il croyait aussi que les personnages devaient évoluer. Il peut arriver de grands événements qui changent totalement la vie des personnages...

V.I. Mais au niveau du langage, du matériau même des échanges entre vos personnages?

G.D. Il y avait certainement là un défi, celui de maîtriser plusieurs langages : lorsque j'écrivais le Ciel par-dessus les toits les chroniques que je lisais à ce moment ressemblaient beaucoup à la langue des vieux Canadiens. Il fallait donc que j'écrive une langue qui suggère une époque, et qu'en écoutant, les gens se sentent transportés dans le temps, qu'ils entendent une langue qui évoque le pays d'il y a trois cents ans. Dans Cap-aux-Sorciers, j'ai voulu évoquer un coin du pays, Charlevoix. J'ai donc introduit beaucoup de termes de navigation. Avec Kanawio (du 15 octobre 1961 au 8 juillet 1962), j'ai voulu faire parler un autre peuple, avec sa propre mentalité. Marie de l'Incarnation avait écrit que c'était une langue très belle, pleine d'images. Je me suis donc obligé de créer en français l'essence même de la langue indienne.

V.I. Il ne s'agissait pas d'une traduction mais de la construction d'une langue indienne avec des matériaux tirés de la langue française, ce qui risquait de dépayser voire de heurter, si l'image que le spectateur se faisait du langage indien ne concordait pas avec la représentation que vous lui offriez.

G.D. On a dérouté un certain nombre de téléspectateurs qui n'étaient pas habitués à entendre parler l'indien comme ça. Quand l'Indien parle français, c'est souvent du jargon. Si nous l'avions fait parler indien on n'aurait rien compris. Il aurait fallu constamment traduire un peu comme dans Shogun. À la radio cela aurait été impossible : il s'agissait de créer une langue neuve. Je ne pouvais pas me servir des régionalismes qui allaient plus tard faire la saveur de la langue de Capaux-Sorciers par exemple.

V.I. Quelle a été l'application pratique des principes énoncés par Robert Choquette, à travers un feuilleton comme Septième nord (29 septembre 1963 - 30 août 1967) par exemple?

G.D. J'ai deux frères médecins qui sont chacun spécialiste d'un domaine particulier et mon père était médecin de famille. Cela me facilitait la tâche. Pour écrire ce Septième nord j'ai demandé à l'un de mes frères de me permettre d'entrer à l'hôpital Notre-Dame. J'ai donc pu assister à des opérations, accompagner des médecins comme si j'étais un interne. Pendant près de sept mois, j'ai vécu la vie d'hôpital, j'ai côtoyé les infirmières, connu le côté humain autant que le côté 
scientifique du milieu hospitalier. J'ai découvert un monde. Je me suis rendu compte que je ne connaissais pas ce qu'était la vie dans un hôpital : avant ce séjour j'avais des idées préconçues qui ne correspondaient pas à la réalité. Fort de mon expérience, j'ai décidé de rendre compte à la fois de la vie des patients et de celle des médecins en évitant cependant d'utiliser un langage scientifique qui serait resté inaccessible au public si je ne l'avais pas modifié. Par ailleurs, pour éviter qu'une personne se reconnaisse à travers l'un des personnages,j'ai procédé un peu comme Grignon lorsqu'il a fait $U n$ homme et son péché : son Séraphin Poudrier est un personnage composite. Les miens aussi. De toute façon c'est l'interaction des personnages qui est devenue la matière même de la série de sorte qu'il y a beaucoup d'éléments que j'ai dô laisser de côté. Cela fait partie, j'imagine, des règles du jeu.

V.I. Des souris et des hommes (1971) passe à juste titre pour l'une des grandes réalisations de Radio-Canada. Malheureusement, bien que votre traduction ait été déposée à la Bibliothèque Nationale, elle n'a pas l'accessibilité qui lui est due, puisqu'il est impossible de la publier pour des raisons juridiques liées au droit international. Pourriez-vous nous parler de cette adaptation de l'ouvre de Steinbeck?

G.D. Depuis Cap-aux-Sorciers, Paul Blouin me parlait de cette œuvre et désirait la réaliser si nous en obtenions les droits. Or Steinbeck avait accordé des droits de traduction exclusifs à un dénommé Michel Duhamel de France. Nous devions donc en principe nous servir de cette traduction. Mais nous avions de sérieux doutes quant aux qualités dramaturgiques de cette traduction que l'on nous imposait en quelque sorte : il ne suffit pas d'appliquer à une langue d'ouvriers agricoles américains, un certain français de Paris pour y saisir toute la vie, toute la sensibilité et le rythme qu'il y a là-dedans. Une traduction littérale nous fait perdre la réalité du personnage : vous vous rappelez de la fameuse réplique de Lenny lorsqu'il aperçoit la première fois la femme de Curly. En anglais c'est «Gosh, she's nice!» Au Québec ça ne prend pas du génie pour traduire «Eh! elle est belle hein!» plutôt que "Ah! mais dites donc ce qu'elle est jolie!» Et il suffit d'imaginer Jacques Godin en train de dire l'une ou l'autre de ces répliques de la traduction de Michel Duhamel pour se faire assez vite une idée de l'échec que la pièce aurait connu! Nous avons donc tout retraduit, tant au niveau de la sensibilité qu'à celui de l'humour'; il y avait une truculence qu'il s'agissait de retrouver à travers les mots.

V.I. Vous avez même été plus loin, car pour recréer l'espace sonore vous avez ajouté au texte une trame vocale qui, bien qu'elle soit jouée par les acteurs est presque inaudible mais contribue fortement à suggérer le paysage ambiant. 
G.D. Robert Choquette nous avait incités à terminer une émission en donnant le goût aux gens de voir ce qui viendrait après. Il croyait aussi que les personnages devaient évoluer. Il peut arriver de grands événements qui changent totalement la vie des personnages...

V.I. Mais au niveau du langage, du matériau même des échanges entre vos personnages?

G.D. Il y avait certainement là un défi, celui de maîtriser plusieurs langages : lorsque j'écrivais le Ciel par-dessus les toits les chroniques que je lisais à ce moment ressemblaient beaucoup à la langue des vieux Canadiens. Il fallait donc que j'écrive une langue qui suggère une époque, et qu'en écoutant, les gens se sentent transportés dans le temps, qu'ils entendent une langue qui évoque le pays d'il y a trois cents ans. Dans Cap-aux-Sorciers, j'ai voulu évoquer un coin du pays, Charlevoix. J'ai donc introduit beaucoup de termes de navigation. Avec Kanawio (du 15 octobre 1961 au 8 juillet 1962), j'ai voulu faire parler un autre peuple, avec sa propre mentalité. Marie de l'Incarnation avait écrit que c'était une langue très belle, pleine d'images. Je me suis donc obligé de créer en français l'essence même de la langue indienne.

V.I. Il ne s'agissait pas d'une traduction mais de la construction d'une langue indienne avec des matériaux tirés de la langue française, ce qui risquait de dépayser voire de heurter, si l'image que le spectateur se faisait du langage indien ne concordait pas avec la représentation que vous lui offriez.

G.D. On a dérouté un certain nombre de téléspectateurs qui n'étaient pas habitués à entendre parler l'indien comme ça. Quand l'Indien parle français, c'est souvent du jargon. Si nous l'avions fait parler indien on n'aurait rien compris. Il aurait fallu constamment traduire un peu comme dans Shogun. À la radio cela aurait été impossible : il s'agissait de créer une langue neuve. Je ne pouvais pas me servir des régionalismes qui allaient plus tard faire la saveur de la langue de Capaux-Sorciers par exemple.

V.I. Quelle a été l'application pratique des principes énoncés par Robert Choquette, à travers un feuilleton comme Septième nord (29 septembre 1963 - 30 août 1967) par exemple?

G.D. J'ai deux frères médecins qui sont chacun spécialiste d'un domaine particulier et mon père était médecin de famille. Cela me facilitait la tâche. Pour écrire ce Septième nord j'ai demandé à l'un de mes frères de me permettre d'entrer à l'hôpital Notre-Dame. J'ai donc pu assister à des opérations, accompagner des médecins comme si j'étais un interne. Pendant près de sept mois, $j$ 'ai vécu la vie d'hôpital, j'ai côtoyé les infirmières, connu le côté humain autant que le côté 
scientifique du milieu hospitalier. J'ai découvert un monde. Je me suis rendu compte que je ne connaissais pas ce qu'était la vie dans un hôpital : avant ce séjour j'avais des idées préconçues qui ne correspondaient pas à la réalité. Fort de mon expérience, j'ai décidé de rendre compte à la fois de la vie des patients et de celle des médecins en évitant cependant d'utiliser un langage scientifique qui serait resté inaccessible au public si je ne l'avais pas modifié. Par ailleurs, pour éviter qu'une personne se reconnaisse à travers l'un des personnages,j'ai procédé un peu comme Grignon lorsqu'il a fait $U n$ homme et son péché : son Séraphin Poudrier est un personnage composite. Les miens aussi. De toute façon c'est l'interaction des personnages qui est devenue la matière même de la série de sorte qu'il y a beaucoup d'éléments que j'ai dû laisser de côté. Cela fait partie, $\mathrm{j}$ 'imagine, des règles du jeu.

V.I. Des souris et des hommes (1971) passe à juste titre pour l'une des grandes réalisations de Radio-Canada. Malheureusement, bien que votre traduction ait été déposée à la Bibliothèque Nationale, elle n'a pas l'accessibilité qui lui est due, puisqu'il est impossible de la publier pour des raisons juridiques liées au droit international. Pourriez-vous nous parler de cette adaptation de l'ouvre de Steinbeck?

G.D. Depuis Cap-aux-Sorciers, Paul Blouin me parlait de cette œuvre et désirait la réaliser si nous en obtenions les droits. Or Steinbeck avait accordé des droits de traduction exclusifs à un dénommé Michel Duhamel de France. Nous devions donc en principe nous servir de cette traduction. Mais nous avions de sérieux doutes quant aux qualités dramaturgiques de cette traduction que l'on nous imposait en quelque sorte : il ne suffit pas d'appliquer à une langue d'ouvriers agricoles américains, un certain français de Paris pour y saisir toute la vie, toute la sensibilité et le rythme qu'il y a là-dedans. Une traduction littérale nous fait perdre la réalité du personnage : vous vous rappelez de la fameuse réplique de Lenny lorsqu'il aperçoit la première fois la femme de Curly. En anglais c'est "Gosh, she's nice!» Au Québec ça ne prend pas du génie pour traduire «Eh! elle est belle hein!» plutôt que «Ah! mais dites donc ce qu'elle est jolie!» Et il suffit d'imaginer Jacques Godin en train de dire l'une ou l'autre de ces répliques de la traduction de Michel Duhamel pour se faire assez vite une idée de l'échec que la pièce aurait connu! Nous avons donc tout retraduit, tant au niveau de la sensibilité qu'à celui de l'humour; il y avait une truculence qu'il s'agissait de retrouver à travers les mots.

V.I. Vous avez même été plus loin, car pour recréer l'espace sonore vous avez ajouté au texte une trame vocale qui, bien qu'elle soit jouée par les acteurs est presque inaudible mais contribue fortement à suggérer le paysage ambiant. 
G.D. Robert Choquette nous avait incités à terminer une émission en donnant le goût aux gens de voir ce qui viendrait après. Il croyait aussi que les personnages devaient évoluer. Il peut arriver de grands événements qui changent totalement la vie des personnages...

V.I. Mais au niveau du langage, du matériau même des échanges entre vos personnages?

G.D. Il y avait certainement là un défi, celui de maîtriser plusieurs langages : lorsque j'écrivais le Ciel par-dessus les toits les chroniques que je lisais à ce moment ressemblaient beaucoup à la langue des vieux Canadiens. Il fallait donc que j'écrive une langue qui suggère une époque, et qu'en écoutant, les gens se sentent transportés dans le temps, qu'ils entendent une langue qui évoque le pays d'il y a trois cents ans. Dans Cap-aux-Sorciers, j'ai voulu évoquer un coin du pays, Charlevoix. J'ai donc introduit beaucoup de termes de navigation. Avec Kanawio (du 15 octobre 1961 au 8 juillet 1962), j'ai voulu faire parler un autre peuple, avec sa propre mentalité. Marie de l'Incarnation avait écrit que c'était une langue très belle, pleine d'images. Je me suis donc obligé de créer en français l'essence même de la langue indienne.

V.I. Il ne s'agissait pas d'une traduction mais de la construction d'une langue indienne avec des matériaux tirés de la langue française, ce qui risquait de dépayser voire de heurter, si l'image que le spectateur se faisait du langage indien ne concordait pas avec la représentation que vous lui offriez.

G.D. On a dérouté un certain nombre de téléspectateurs qui n'étaient pas habitués à entendre parler l'indien comme ça. Quand l'Indien parle français, c'est souvent du jargon. Si nous l'avions fait parler indien on n'aurait rien compris. Il aurait fallu constamment traduire un peu comme dans Shogun. À la radio cela aurait été impossible : il s'agissait de créer une langue neuve. Je ne pouvais pas me servir des régionalismes qui allaient plus tard faire la saveur de la langue de Capaux-Sorciers par exemple.

V.I. Quelle a été l'application pratique des principes énoncés par Robert Choquette, à travers un feuilleton comme Septième nord (29 septembre 1963 - 30 août 1967) par exemple?

G.D. J'ai deux frèrés médecins qui sont chacun spécialiste d'un domaine particulier et mon père était médecin de famille. Cela me facilitait la tâche. Pour écrire ce Septième nord j'ai demandé à l'un de mes frères de me permettre d'entrer à l'hôpital Notre-Dame. J'ai donc pu assister à des opérations, accompagner des médecins comme si j'étais un interne. Pendant près de sept mois, j'ai vécu la vie d'hôpital, j'ai côtoyé les infirmières, connu le côté humain autant que le côté 
scientifique du milieu hospitalier. J'ai découvert un monde. Je me suis rendu compte que je ne connaissais pas ce qu'était la vie dans un hôpital : avant ce séjour j'avais des idées préconçues qui ne correspondaient pas à la réalité. Fort de mon expérience, j'ai décidé de rendre compte à la fois de la vie des patients et de celle des médecins en évitant cependant d'utiliser un langage scientifique qui serait resté inaccessible au public si je ne l'avais pas modifié. Par ailleurs, pour éviter qu'une personne se reconnaisse à travers l'un des personnages,j'ai procédé un peu comme Grignon lorsqu'il a fait $U n$ homme et son péché : son Séraphin Poudrier est un personnage composite. Les miens aussi. De toute façon c'est l'interaction des personnages qui est devenue la matière même de la série de sorte qu'il y a beaucoup d'éléments que j'ai dû laisser de côté. Cela fait partie, j'imagine, des règles du jeu.

V.I. Des souris et des hommes (1971) passe à juste titre pour l'une des grandes réalisations de Radio-Canada. Malheureusement, bien que votre traduction ait été déposée à la Bibliothèque Nationale, elle n'a pas l'accessibilité qui lui est due, puisqu'il est impossible de la publier pour des raisons juridiques liées au droit international. Pourriez-vous nous parler de cette adaptation de l'œuvre de Steinbeck?

G.D. Depuis Cap-aux-Sorciers, Paul Blouin me parlait de cette ouvre et désirait la réaliser si nous en obtenions les droits. Or Steinbeck avait accordé des droits de traduction exclusifs à un dénommé Michel Duhamel de France. Nous devions donc en principe nous servir de cette traduction. Mais nous avions de sérieux doutes quant aux qualités dramaturgiques de cette traduction que l'on nous imposait en quelque sorte : il ne suffit pas d'appliquer à une langue d'ouvriers agricoles américains, un certain français de Paris pour y saisir toute la vie, toute la sensibilité et le rythme qu'il y a là-dedans. Une traduction littérale nous fait perdre la réalité du personnage : vous vous rappelez de la fameuse réplique de Lenny lorsqu'il aperçoit la première fois la femme de Curly. En anglais c'est «Gosh, she's nice!» Au Québec ça ne prend pas du génie pour traduire «Eh! elle est belle hein!» plutôt que «Ah! mais dites donc ce qu'elle est jolie!» Et il suffit d'imaginer Jacques Godin en train de dire l'une ou l'autre de ces répliques de la traduction de Michel Duhamel pour se faire assez vite une idée de l'échec que la pièce aurait connu! Nous avons donc tout retraduit, tant au niveau de la sensibilité qu'à celui de l'humour; il y avait une truculence qu'il s'agissait de retrouver à travers les mots.

V.I. Vous avez même été plus loin, car pour recréer l'espace sonore vous avez ajouté au texte une trame vocale qui, bien qu'elle soit jouée par les acteurs est presque inaudible mais contribue fortement à suggérer le paysage ambiant. 
G.D. Blouin avait le souci de la vérité : lorsque Lenny caresse la femme de Curly dans la grange, un dimanche après-midi ensoleillé, pendant que les gars jouent aux fers, l'auteur mentionne simplement («Ding», un fer a frappé le poteau). Il fallait qu'on les entende (même s'ils n'étaient pas vus des spectateurs et si l'auteur n'avait rien dit à leur sujet), que soit suggérée cette vie de dimanche après-midi, dans la cour, à cent pieds de la grange. Nous sommes donc allés à Farnham dans un club voir des gars qui jouaient aux fers. Accoudés pendant deux heures à la clôture; on les a observés. Je connaissais les règles du jeu. Je l'avais déjà pratiqué. Mais je n'aurais pas pu décrire la scène si je ne m'étais pas replongé dans l'ambiance : qu'est-ce qui se passe entre les joueurs? quelles réflexions font-ils? quel est le vécu qui rend la scène authentique? Paul Blouin a même engagé l'un des joueurs, m'a demandé d'écrire des scènes avec deux joueurs, d'autres avec un plus grand nombre, ce que j'ai fait à partir de mes observations et des règlements que l'on m'avait remis! Tout ce travail pour une courte scène où l'on entendait des voix indistinctes. Mais ça faisait vrai, ça faisait vivant.

V.I. Très vivant en effet. C'est d'ailleurs votre principale qualité. Certaines de vos ouvres ont germé d'écrits dont on n'aurait pas soupçonné un tel épanouissement : des textes à caractère légal ou administratif par exemple.

G.D. Dans le cas des Forges du St-Maurice, je partais à zéro ou presque : je suis allé sur les lieux en plein hiver, c'était quelque part derrière TroisRivières et les bâtiments n'avaient pas encore été reconstitués, les familles commençaient à peine. C'était un endroit sauvage en février ou mars, battu par un grand vent et je me suis dit que ce n'était pas possible d'avoir affronté des conditions de température pareilles sans qu'il y ait eu des drames et dés comédies à foison! D'autre part j'ai mis la main, grâce à Jacques Lacourcière, sur un document inestimable qui m'a permis de créer mes personnages. À la suite d'une faillite, l'enquêteur, monsieur Estèbes, avait fait le portrait des employés, sans complaisance, dans le style : un tel, tel âge, est habile mais brouillon et ivrogne, batailleur. Il faut le tenir d'une main ferme.. J'avais le portrait de chacun des ouvriers. Par ailleurs, j'ai parcouru la région. Ainsi, j'ai visité une petite forge à Sainte-Anne-de-la-Pérade. Il fallait que je voie les ouvriers en sueur faire une coulée, que je les observe sur place pour pouvoir parler d'eux convenablement.

V.I. Parlez-nous de votre expérience cinématographique, plus particulièrement des Ordres (1974), avec Michel Brault...

G.D. Michel Brault avait recueilli lors de la «crise d'octobre 1970» autour de cinquante témoignages, partout au Québec, beaucoup au Lac Saint-Jean, à Québec, à Montréal, dans toutes les couches de la société 
mais il ne pouvait pas présenter cinquante-deux témoignages comme ça, dans un film. À la Société de développement de l'industrie cinématographique du Canada, on lui a suggéré de s'entendre avec un scénariste. À partir des témoignages qu'il avait glanés, Brault entrevoyait déjà cinq ou six personnages dont un médecin, un chauffeur de taxi syndicaliste, sa femme, un ouvrier spécialisé dont la femme était serveuse de restaurant. À partir de ce canevas, aidé de cinq ou six comédiens, il était prêt à se jeter à l'eau! Je ne dis pas cela avec ironie, Brault aime l'aventure, l'improvisation dans le bon sens du mot. Mais les commanditaires lui disaient : les comédiens risquent de ne pas être capables de traduire les témoignages qui sont là.

Nous avons donc consacré une grosse veillée, sinon une nuit à parler de ses personnages, à tenter de les définir : quel était leur tempérament? quelle instruction avaient-ils? comment parlaient-ils? Une fois que tout cela a été placé, je me suis mis à écrire à tord-cou (le tournage commençait dans quinze jours), à tâcher de penser comment chacun vivait, à prendre les témoignages et les mettre dans la bouche, dans la vie des personnages. Â mesure que j'écrivais, c'était l'épine dorsale du scénario qui se formait et le texte à peine terminé, le tournage a commencé. Au point où à un certain moment, il y a eu un enchaînement à faire, une lettre à écrire que j'ai dû faire immédiatement, sur les lieux même du tournage!

V.I. Guy Dufresne, en vous remerciant de cette entrevue, pourriez-vous nous parler de vos projets en cours de diffusion ou de réalisation?

G.D. En premier lieu, un court métrage intitulé C'est pour Mathieu, qui a été réalisé par un jeune cinéaste, Dominique Boisvert. Il s'agit d'une émission d'une demi-heure produite par l'Institut québécois du cinéma et Radio-Québec : une nuit d'incendie, une jeune femme qui a un enfant est obligée de "paqueter» en vitesse pour quitter la maison en flammes. Certains des voisins sont absents. L'un d'entre eux, toutefois, qui a l'habitude de surveiller son enfant, est un personnage original, inventif, je dirais presque un mythomane. Il va tout raconter sinon imaginer ce qui s'est passé : les causes du désastre, ses incidences. En fait, les versions peuvent toutes être vraisemblables! C'est une comédie dramatique qui va se terminer de manière inattendue grâce à un événement que l'on m'a raconté et que j'ai pu introduire dans le récit.

Je travaille à une sorte de «thriller» qui sera réalisé par Louis-Georges Carrier : c'est une série intitulée Aéroport à laquelle participent la France, la Belgique, la Suisse et le Québec. L'action se passe dans le Grand-Nord, à La Grande, dans la Baie James qui est un endroit où, à cause des distances que l'on imagine difficilement, on se sert beaucoup de l'avion. Il fallait trouver un suspense : voilà c'est fait. 
Enfin les autres projets sont à l'état d'ébauche. Je préfère ne pas en parler tout de suite! 